\title{
Primary Leptomeningeal PNETs Mimicking TB Meningitis: A Case Report and Literature Review
}

\author{
I-Han HSIAO,Chao-Hsuan CHEN, Han-Chung LEE, Der-Yang CHO \\ China Medical University Hospital, Department of Neurosurgery, Taichung, Taiwan
}

\section{ABSTRACT}

Primitive neuroectodermal tumors (PNETs) are easily detected, and the diagnosis made by neuroradiologic imaging. Leptomeningeal seeding is a common complication and carries a poor prognosis. Primary leptomeningeal PNETs are rarer and difficult to diagnose on magnetic resonance imaging (MRI) or cerebrospinal fluid (CSF) studies. We encountered a rare case of primary leptomeningeal primitive neuroectodermal tumor without intracranial mass lesion which was diagnosed by spinal lesion biopsy. This 25-year-old woman presented with worsening headaches and progressive visual impairment.The initial diagnosis was tubercle bacillus (TB) meningitis, and the final diagnosis of primary leptomeningeal PNET was made by spinal biopsy. We present this illustrative case, review previous cases in the literature, and suggest that diagnosis is considered in mildly affected patients with similar symptoms.

KEYWORDS: Neuroectodermal tumors, CD99 antigen, Human, Magnetic resonance imaging, Meningitis

\section{INTRODUCTION}

$\mathrm{P}$ rimitive neuroectodermal tumors (PNETs) of the central nervous system (CNS) are highly malignant neoplasms that occur predominantly in children. They are typically circumscribed and carry a risk of spreading to the leptomeninges. Primary leptomeningeal PNETs are rarer and can mimic meningitis on neuro-imaging or cerebrospinal fluid (CSF) studies.

We report a case of a 25 -year-old female patient with primary disseminated PNET that produced elevated intracranial pressure without an identifiable mass. The final diagnosis was made through results of a spinal biopsy.

\section{CASE REPORT}

A 25-year-old previously healthy female patient had a 5-month history of episodic headaches and vomiting, with progressively blurred vision. Her symptoms occurred mostly in the early morning hours. Physical examination revealed bilateral papilledema with declining visual acuity. There were no obvious motor weakness or paresthesia. Magnetic resonance imaging (MRI) of the brain, with the addition of gadoliniumdiethylinium-diethylene triamine pentaacetic acid (Gd-DTPA) revealed diffuse linear leptomeningeal enhancement over the cerebral sulcus and bilateral sylvian fissure, without an intraparenchymal mass (Figure 1A-C). Evaluation of the CSF from lumbar puncture revealed an initial pressure of 270 $\mathrm{mmH}_{2} \mathrm{O}$, and CSF chemistry showed low glucose $(26 \mathrm{mg} / \mathrm{dL})$ and high protein (198 mg/dL) levels, but normal cell counts. The suspected diagnosis was chronic meningitis due to tuberculosis or cryptococcal infection. CSF cytology, culture, Indian ink test, autoimmune profile, and TB polymerase chain reaction (PCR) were all negative.

The patient was treated with anti-tuberculosis agents and steroids for about one month, but her symptoms persisted. Lumbar puncture repeated on three occasions relieved her headache and nausea, as well as her blurred vision. CSF results remained unchanged. A right ventriculoperitoneal shunt was placed, and her symptoms gradually improved. The results of CSF studies from ventricular taps were similar to those after lumbar puncture, and cytologic results remained negative. 
Whole-spine MRI with Gd-DTPA revealed diffuse leptomeningeal enhancement through the cervicothoracolumbar spine (Figure 1D, E). In addition, a small nodule was detected at the left L4 root (Figure 1F). L4 laminectomy was performed, and a tumor infiltrating the epidural, perineural and arachnoid membrane was found. Pathology reports revealed small blue round cell tumor of L4; all sections were involved (Figure 3A, B).

Undifferentiated tumor cells with round nuclei and scanty cytoplasm were seen discohesively invading the arachnoid membrane and connective tissues. Immunohistochemical tests were positive for synaptophysin, negative for CD99, lectin Lens culinaris agglutinin (LCA), and terminal deoxynucleotidyl transferase (TdT). A diagnosis of a CNS primary leptomeningeal PNET was made.

The patient received chemotherapy with vincristine, cisplatin, etoposide, and cyclophosphamide. After six months, the leptomeningeal enhancement disappeared in the following brain (Figure 2A, B) and L-spine MRI (Figure 2C). She was followed up for 2 years with no recurrence.

\section{DISCUSSION}

The first mention of primary diffuse leptomeningeal tumors (PDL) without systemic or CNS intraparenchymal lesion can be traced back to the 1920s, when a case of "sarcomatosis" of the meninges was described by Connor and Cushing (1). Subsequently, more accurate terms, for example, PDL gliomatosis or PDL ependymoblastoma, were developed for this lesion,

PNETs are highly malignant CNS neoplasms that arise from pluripotent neural crest cells. The primary tumor typically grows as a circumscribed and frequently cystic mass. In children and young adults, these lesions are frequently located at the posterior fossa and are easily detected with neuroradiographic imaging. PNETs have the highest rate of leptomeningeal involvement at the time of diagnosis in children (2), and supratentorial PNETs with leptomeningeal spread have a poor prognosis. Instances of peripheral PNET/Ewing sarcomas arising intracranially have been reported; however,
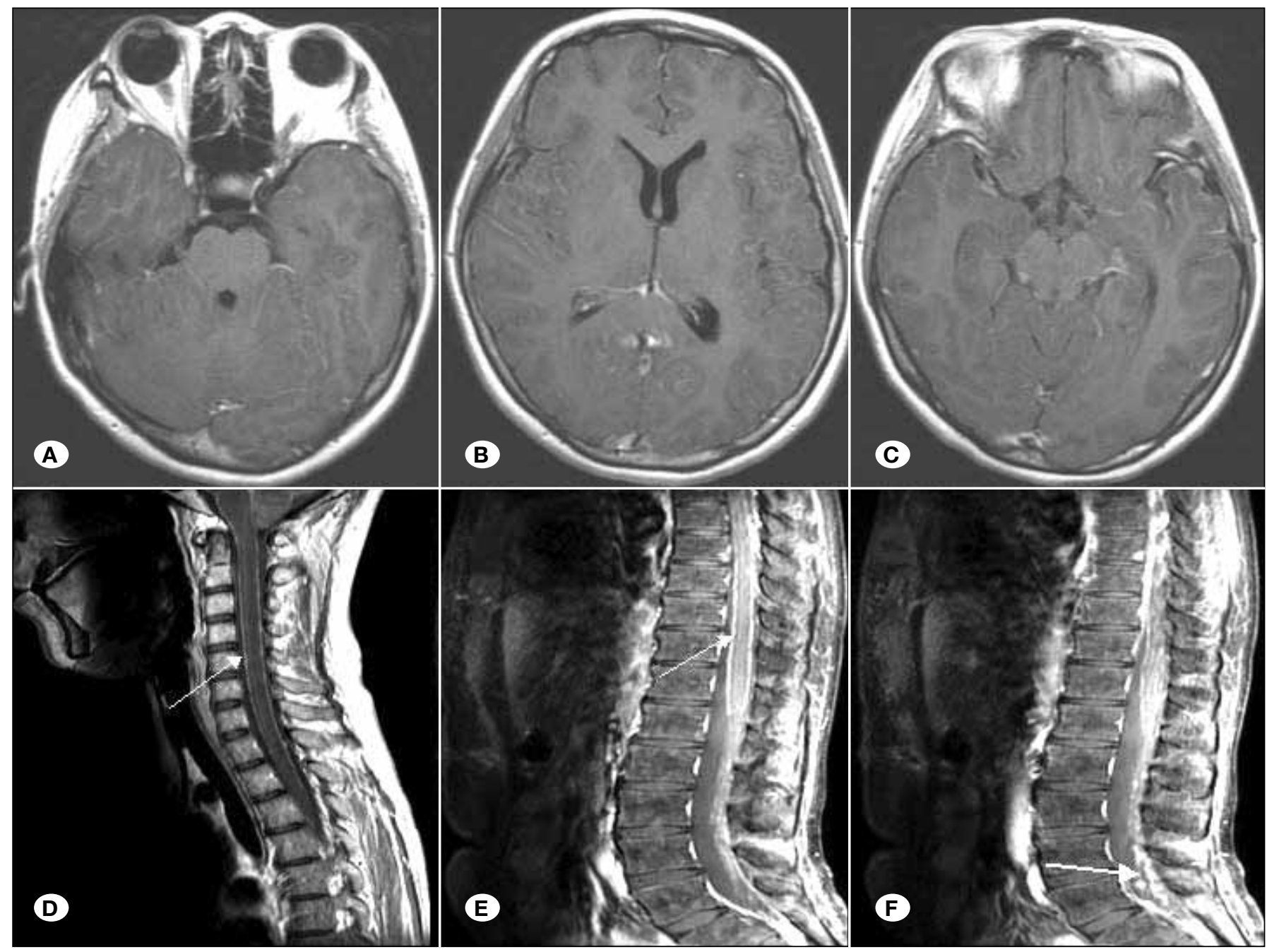

Figure 1: A-C) Axial T1-weighted MRI with Gd-DTPA showing diffuse linear leptomeningeal enhancement. D,E) Whole spine MRI with Gd-DTPA also shows diffuse leptomeningeal enhancement (thin white arrow). F) A nodule over the L4 root with epidural soft tissue enhancement (thick white arrow). 


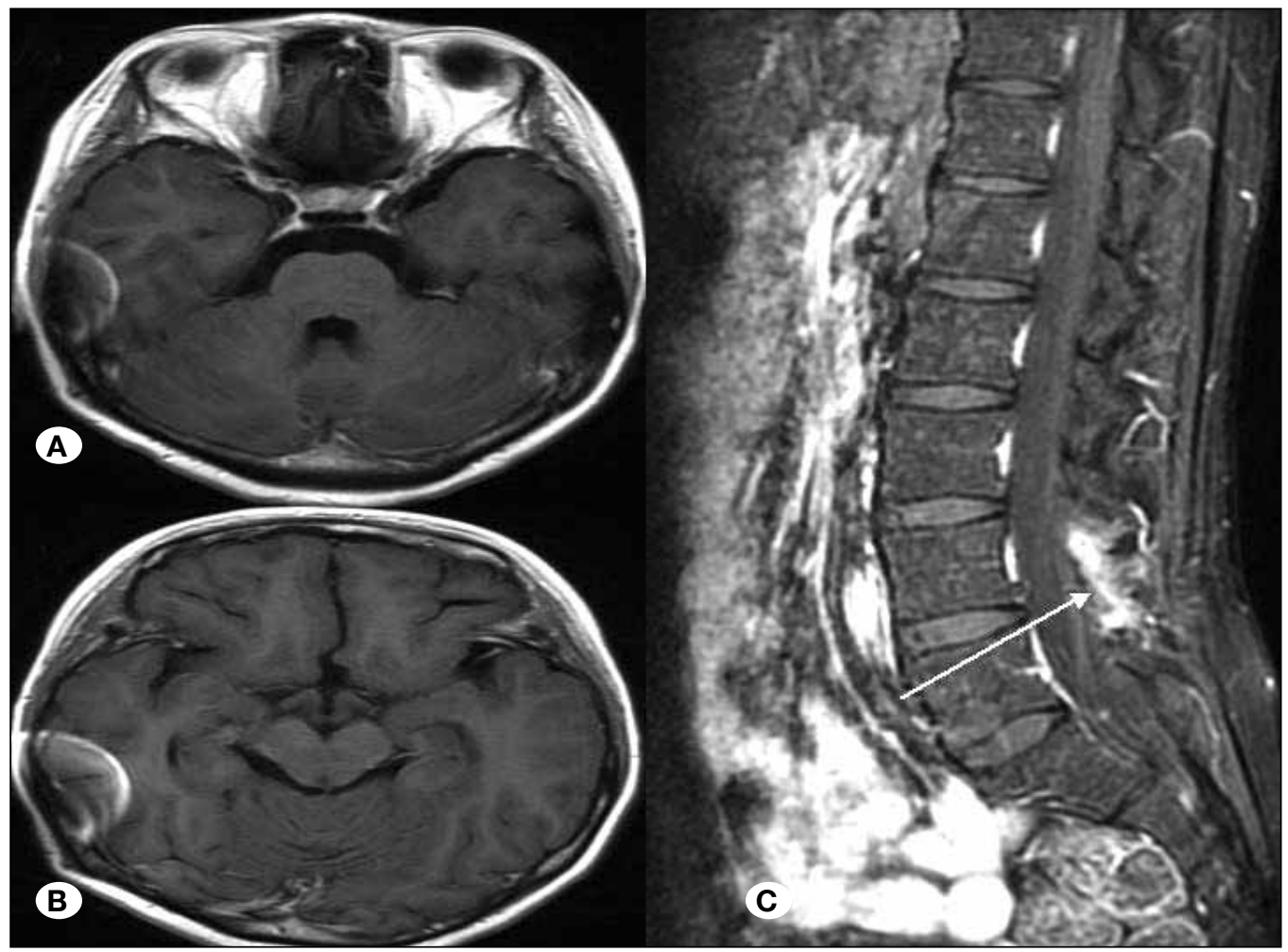

Figure 2: A,B) Axial T1-weighted MRI with Gd-DTPA: The previous leptomeningeal enhancement has disappeared. C) Lumbar spine MRI with Gd-DTPA: previous leptomeningeal enhancement and nodule have disappeared (white arrow). these sarcomas reveal a strong expression of CD99, which is recognized by anti-CD99 monoclonal (O13) antibodies (3). In contrast, CD99 antigen is negative in cases of central PNET, as with our patient.

The origin of primary leptomeningeal PNET is unknown, although it has been suggested that they arise from heterotopic glial nests in the arachnoid space (4). Because the diagnosis of primary leptomeningeal PNET is difficult to make, treatment is often delayed. It's believed that MR imaging and CSF studies are the best diagnostic tools for detecting leptomeningeal disease, including infectious disease and neoplasms.

In patients with CNS leptomeningeal enhancement on contrastenhanced T1 weighted MRI without a true mass lesion often lead the diagnosis to meningitis, especially in young adults without systemic disease, as was the case with our patient. Since tuberculous meningitis usually causes accumulation of gelatinous exudates in the cerebral cisterns on MR imaging, and is life threatening with delay treatment, early empirical anti-TB therapy is often prescribed. CSF biochemistry often shows low glucose levels containing high amounts of protein, which can appear in both infectious disease and neoplasms. Therefore, the presence of tumor cells in the CSF is important for making the diagnosis. However, the optimal site for CSF sampling has been a point of debate. Some authors have reported that the malignant cells were detected at a significantly higher rate when lumbar puncture was used than when intracranial CSF was obtained (6). Other authors have reported that intracranial CSF sampling is more sensitive and also can detect dissemination of disease earlier (7). Although the results of cytologic studies were only been able to confirm a positive $\mathrm{MRI}$ in $70 \%$ of cases, $40 \%$ of patients found to have neoplastic meningismus at autopsy had negative CSF cytological results premortem. However, these series involved patients with a known CNS tumor with CSF seeding. For leptomeningeal tumors, there were no reports or information about ideal CSF sampling sites.

Few reports of leptomeningeal PNETs without evidence of primary tumors have been made (Table I) (4,8-13). These cases also occurred mainly in children and young adults, and patients usually presented with symptoms of chronic meningitis, such as headache and vomiting, which may be related to increased intracranial pressure. Three cases were diagnosed by postmortem brain autopsy, six by CSF sampling, and the remaining three by brain biopsy by craniotomy.

To the best of our knowledge, Gurkanlar et al. published the first case of primary spinal PNET at the Th12-L1 and L5-S1 levels associated with hydrocephalus (5). The case we report here might be the second instance where primary leptomeningeal PNET was diagnosed by a spinal biopsy and was also associated with hydrocephalus. Evaluation of the biopsy specimens, including epidural soft tissue, perineural tumors, and arachnoid membrane, revealed small, blue, round cells. Either central or peripheral PNET could have been suspected but the negative study with CD99 proved the diagnosis of central PNET. In most previous case reports, patients survived only from 2 weeks to 3 years after the diagnosis was made. Most lived less than 18 months after leptomeningeal PNET was diagnosed. Survival was shorter than formal PNET survival periods and even though leptomeningeal seeding carried a poor prognosis, making a correct diagnosis early, followed by appropriate treatment, may help extend the patient's life. 


\section{CONCLUSION}

Primary leptomeningeal PNET is difficult to diagnose and as a result patients often have a poor prognosis and a short survival time. Therefore, when children or young adults present with symptoms including hydrocephalus occurring and signs of chronic meningitis and failed treatment with empiric antibiotics, we suggest a thorough workup, including brain and wholespine MRls with repeated CSF sampling, beginning with the less-invasive lumbar puncture. If meningeal enhancement is strong but CSF cytology cultures are all negative, a ventricular tap is needed and meningeal biopsy, either cerebral or spinal, should then be considered.

Table I: Reports of Leptomeningeal PNETs without Evidence of Primary Tumors

\begin{tabular}{|c|c|c|c|c|c|}
\hline Study & Year & $\begin{array}{c}\text { Age and symptoms at } \\
\text { presentation }\end{array}$ & Diagnosis & Diagnostic tool & Survival \\
\hline Bailey & 1985 & $\begin{array}{l}\text { 19-year-old female, } \\
\text { meningismus }\end{array}$ & PNETS & Brain autopsy & 18 months \\
\hline Wada & 1986 & $\begin{array}{l}\text { 17-year-old female, } \\
\text { headache, convulsions }\end{array}$ & ependymoblastoma & Brain autopsy & 3 months \\
\hline Szpak & 1995 & 19-year-old female & PNETs & Brain autopsy & 18 months \\
\hline Ebinger & 2000 & Two 7-year-old males & PNETs & Biopsy & \\
\hline Begemann & 2003 & 8-year-old male & PNETs & CSF studies & 3 years \\
\hline
\end{tabular}

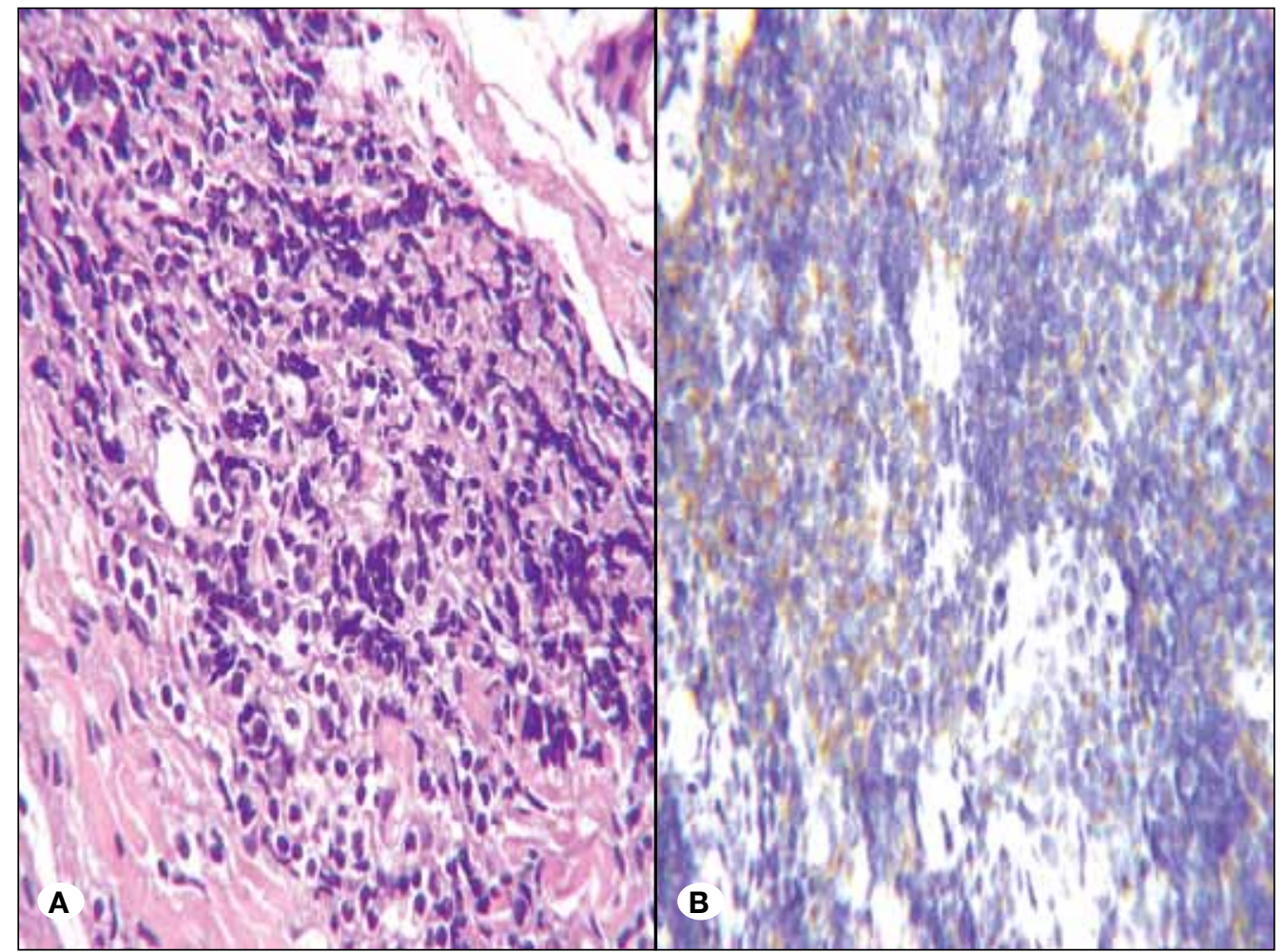

Figure 3: A) Microscopically, the specimen shows a picture of small blue round cell tumor in the tissue fragments of epidural space adjacent to L4 vertebra, manifested as undifferentiated tumor cells with regularly round nuclei and scanty cytoplasm discohesively infiltrating the arachnoid and connective tissue. B) The immunohistochemical study revealed positive staining of synaptophysin and negative of CD99, LCA and TdT. 


\section{REFERENCES}

1. Bailey P, Robitaille Y: Primary diffuse leptomeningeal gliomatosis. Can J Neurol Sci 12:278-281, 1985

2. Begemann M, Lyden D, Rosenblum MK, Lis E, Wolden S, Antunes NL, Dunkel IJ: Primary leptomeningeal primitive neuroectodermal tumor. J Neurooncol 63:299-303, 2003

3. Connor CL, Cushing $\mathrm{H}$ : Diffuse tumors of leptomeninges. Two cases in which the process was revealed only by the microscope. Arch Pathol 3:374-392, 1927

4. Ebinger F, Bruhl K, Gutjahr P: Early diffuse leptomeningeal primitive neuroectodermal tumors can escape detection by magnetic resonance imaging. Childs Nerv Syst 16:398-401, 2000

5. Gurkanlar D, Korkmaz E, Gurler IE, Gokhan G, Kazan S: Multilevel primary intraspinal PNETs in an infant associated with hydrocephalus. Turk Neurosurg 20(1):82-85, 2010

6. Jennings MT, Slatkin N, D'Angelo M, Ketonen L, Johnson MD, Rosenblum M, Creasy J, Tulipan N, Walker R: Neoplastic meningitis as the presentation of occult primitive neuroectodermal tumors. J Child Neurol 8: 306-312, 1993

7. Maryam F, Amar G, James MB, Andrew W, Stephen JT: Comparison of CSF cytology and spinal magnetic resonance imaging in the detection of leptomeningeal disease in pediatric medulloblastoma or primitive neuroectodermal tumor. J Clin Oncol 17: 3234-3237, 1999
8. Packer RJ, Siegel KR, Sutton LN, Litmann P, Bruce DA, Schut $\mathrm{L}$ : Leptomeningeal dissemination of primary central nervous system tumors of childhood. Ann Neurol 18:217-221, 1985

9. Suman R, Santosh V, Anandh BA: Primary leptomeningeal medulloblastoma. Pediatr Neurosurg 43:544-545, 2007

10. Szpak GM, Papierz W, Liberski PP, Kulczycki J, KrystWidzgowska T, Dymecki J: Primitive neuroectodermal tumor (PNET). A case report. Folia Neuropathol 33:35-40, 1995

11. Terterov S, Krieger MD, Bowen I, McComb JG: Evaluation of intracranial cerebrospinal fluid cytology in staging pediatric medulloblastomas. supratentorial primitive neuroectodermal tumors, and ependymomas. J Neurosurg Pediatr 6:131-136, 2010

12. Wada C, Kurata A, Hirose R, Tazaki Y, Kan S, Ishihara Y, Kameya T: Primary leptomeningeal ependymoblastoma. Case report. J Neurosurg 64:968-973, 1986

13. Weidner N, Tjoe J: Immunohistochemical profile of monoclonal antibody 013: Antibody that recognizes glycoprotein p30/32 MIC2 and is useful in diagnosis Ewing's sarcoma and peripheral neuroepithelioma. Am J Surg Pathol 18:486-494, 1994 